\title{
Formation-based approach for multi-robot cooperative manipulation based on optimal control design
}

\author{
Dominik Sieber, Frederik Deroo and Sandra Hirche
}

\begin{abstract}
Cooperative manipulation, where several robots collaboratively transport an object, poses a great challenge in robotics. In order to avoid object deformations in cooperative manipulation, formation rigidity of the robots is desired. This work proposes a novel linear state feedback controller that combines both optimal goal regulation and a relaxed form of the formation rigidity constraint, exploiting an underlying distributed impedance control scheme. Since the presented control design problem is in a biquadratic LQR-like form, we present an iterative design algorithm to compute the controller. As an intermediate result, an approximated state-space model of an interconnected robot system is derived. The controller design approach is evaluated in a full-scale multi-robot experiment.
\end{abstract}

\section{INTRODUCTION}

The cooperation and interaction of several mobile manipulators to achieve a common task is a popular research topic in recent years. A specific problem in the area is the collaborative manipulation of objects. In this paper, we investigate the prototypical task of several physically cooperating manipulators, which collaboratively transport an object from an initial to a final configuration. In this setup, each manipulator is impedance-controlled and can thus react compliantly to its environment. The contact environment can be represented by the object, an external force, or the other cooperating manipulator. The focus of this paper is on the design of an optimal control scheme for an interconnected multi-robot system integrating a rigidity constraint into the objective. The control scheme requires two main features: First, goal regulation in order to drive the system to a final configuration, preferably in an optimal fashion and second, coordination such that the overall system with distributed impedances does not damage the transported object.

A related approach to this problem consists of distributing the impedances to multiple cooperating manipulators in closed contact which extends the capabilities of the robot team in transportation and contact tasks [1], [2]. Their method requires a path planning of individual trajectories for each manipulator by considering grasp points and the object geometry. However, these developed plans are very sensitive to the initial configurations and to the model accuracy. Also loosely related are methods in formation control of mobile robots, where no manipulation in physical cooperation is considered. [3]-[6]. In [3] a formation control problem is presented for agents with integrator dynamics using a biquadratic, positive definite function, which they employ

All authors are with the Institute for Information-Oriented Control, Department of Electrical Engineering and Information Technology, Technische Universität München, D-80290 Munich, Germany. \{dominik.sieber, fred.deroo, hirche\}@tum.de for the leader-follower approach in mobile robots. Using the rigidity constraint in the control design for interaction with a leader-follower network is presented in [4]. Controlling and maintaining triangular formations for mobile autonomous agents is studied in [5], [6]. Keeping the formation while moving in an optimal fashion to a goal configuration is one of the main challenges which has not yet been addressed for multi-robot cooperative manipulation in the known literature. In this context the formation relates to rigidity constraints, which are necessary to avoid unbounded internal forces in the closed kinematic chain. For optimal control of linear systems and quadratic cost functionals, the linear quadratic regulator (LQR) approach is widely employed [7]. It enables a consistent and transparent multivariable control system design for linear systems. Weighting matrices in the cost term are used for different control goals and its solution results in a state feedback control law.

The main contribution of this paper is a suboptimal LQRlike control law for physically cooperating manipulators transporting a rigid object that regulates the robotic system in an optimal fashion to a final configuration while maintaining its formation. To achieve the LQR-like control law, we formulate an optimal control problem which combines the classical quadratic cost function with a relaxed formation (rigidity) constraint in terms of an additional biquadratic penalty term. This relaxed rigidity constraint is justified by the use of impedance control, by which minor deviations from the rigidity constraint result in tolerable object stress. Because of the biquadratic term the LQR problem cannot be solved using standard methods, we propose an iterative descent method inspired by [8] and [9]. As an intermediate step in the controller design, we introduce an approximated state-space model for physically cooperating multi-robotteams. Our proposed controller is evaluated in experiments and its benefit for cooperative tasks is shown. For a theoretical treatment using numerical investigations of iterative control design under relaxed rigidity we refer to [10].

The remainder of this paper is structured as follows. Section II describes formally the cooperative multi-robot team problem considered in this work, and formulates the control goals. The proposed controller design is presented in III which relaxes the rigid formation constraint in a biquadratic cost term, and which iteratively determines the optimal feedback controller design with the iterative QuasiNewton BFGS method. An evaluation of the approach in a multi-robot experiment is shown in IV.

Notation: Bold symbols denote vectors. The vectorization of a matrix is denoted by $\operatorname{vec}(\cdot)$, the inverse operation of 


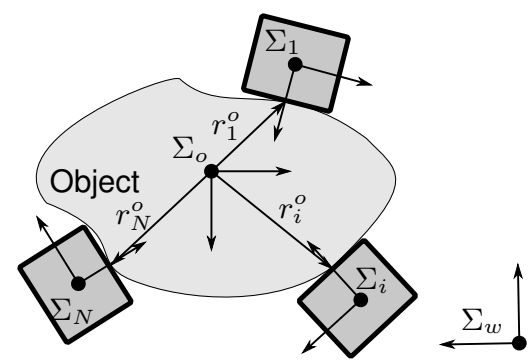

Fig. 1. Illustration of the coordinate frames robots, object, and world

reshaping a vector into a matrix is denoted by mat $(\cdot)$. The identity matrix of dimension $n$ is signified by $I_{n}$. The zero matrix of dimension $m \times n$ is denoted by $0_{m \times n}$.

\section{RIGIDITY-RELAXED MULTI-ROBOT SYSTEM}

This section introduces a state-space model for interconnected cooperative multi-robot teams, which is employed for the control design. The schematics of the cooperating mobile manipulators is depicted in Fig. 1 with the attached coordinate systems. For the $i$-th manipulator, position and orientation of the end-effector frame $\Sigma_{i}$ is expressed in a world coordinate system $\Sigma_{w}$. An object-centered frame $\Sigma_{o}$ is aligned to the principal axes of the object. The matrix $R_{o}^{i}$ describes the rotation of $\Sigma_{i}$ relative to $\Sigma_{o}$.

\section{A. Multi-robot cooperation - a state space model}

Consider a cooperative team of interacting robots $i=1, \ldots, N$, each one evolving according to the inverse dynamic feedback-linearized impedance control.

1) Impedance Control Scheme: Since an impedance control enforces a compliance of the manipulator to its environment, it is widely used in manipulation tasks. Accordingly, the system dynamics of one manipulator evolve according to

$$
M_{i} \ddot{\boldsymbol{\xi}}_{i}+D_{i}\left(\dot{\boldsymbol{\xi}}_{\boldsymbol{i}}-\dot{\boldsymbol{\xi}}_{i, d}\right)+K_{i}\left(\boldsymbol{\xi}_{\boldsymbol{i}}-\boldsymbol{\xi}_{i, d}\right)=\boldsymbol{f}_{i, d}-\boldsymbol{f}_{i} .
$$

Here, $\boldsymbol{\xi}_{i} \in \mathbb{R}^{n}, i=1, \ldots, N$ is the Cartesian position of the $i$-th manipulator, its time derivatives $\dot{\boldsymbol{\xi}}_{i}$ and $\ddot{\boldsymbol{\xi}}_{i}$ are velocity and acceleration, respectively. The positive definite matrices $M_{i}, D_{i}$, and $K_{i} \in \mathbb{R}^{n \times n}$ are the inertia, damping, and stiffness constituting the motion control scheme w.r.t. the control inputs: The desired force $\boldsymbol{f}_{i, d}$ and the desired velocity $\dot{\boldsymbol{\xi}}_{i, d}$, where the desired position $\boldsymbol{\xi}_{i, d}$ is inferred via integration. Further, $\boldsymbol{f}_{i} \in \mathbb{R}^{n}$ denotes the resulting force. For a single robotic manipulator, this force $\boldsymbol{f}_{i}$ arises from contact with the environment. However, since cooperating manipulators are in contact through the object, an internal force among the physically cooperating manipulators is present.

2) Partition of $\boldsymbol{f}_{i}$ : In general, we consider a measured end-effector force $\boldsymbol{f}_{i}^{*}$ composed of rigid-body dynamics $\boldsymbol{f}_{\text {motion }}$, external force $\boldsymbol{f}_{\text {ext }}$, and internal force $\boldsymbol{f}_{i \text {,int }}$, i.e.

$$
\boldsymbol{f}_{i}^{*}=\frac{1}{N}\left(\boldsymbol{f}_{\text {motion }}+\boldsymbol{f}_{\text {ext }}\right)+\boldsymbol{f}_{i, \text { int }} .
$$

The resulting force $\boldsymbol{f}_{i}$ in (1) can be unequal to the measured force $\boldsymbol{f}_{i}^{*}$, in our case we set $\boldsymbol{f}_{i}=\boldsymbol{f}_{i}^{*}-\frac{1}{N} \boldsymbol{f}_{\text {motion }}$.
Hence, the rigid body dynamics $\boldsymbol{f}_{\text {motion }}$ is suppressed in the impedance (1). This is reasonable, since a compliance resultant from the dynamics of the object leads to a permanent undesired position deviation of the multi-robot team, e.g. the object mass pulling down the manipulators.

Assumption 1: We assume that all resulting forces are equally distributed among the manipulators; the factor $\frac{1}{N}$ accounts for that. This approximation demands equal impedance parameters $M_{i}, D_{i}$, and $K_{i}$ for all manipulators.

The resulting object force $\boldsymbol{f}_{\text {motion }}$ arises from Newton's second law of motion and it can generally be expressed as

$$
\boldsymbol{f}_{\text {motion }}=M_{o} \ddot{\boldsymbol{\xi}}_{o}+f_{o}\left(\boldsymbol{\xi}_{o}, \dot{\boldsymbol{\xi}}_{o}\right),
$$

where $\boldsymbol{\xi}_{o}$ is the position of the object with its inertia $M_{o}$. For this paper, we assume $M_{o}$ and $f_{o}$ to be known accurately and thus the impedance control is independent from the object dynamics. The external force $\boldsymbol{f}_{\text {ext }}$ can originate from an undesired obstacle or from a desired physical human input.

To calculate the virtual linkage model of internal forces, all measured end-effector forces are aggregated: $\boldsymbol{f}^{o}=\left[\boldsymbol{f}_{1}^{o^{T}}, \ldots, \boldsymbol{f}_{N}^{o^{T}}\right]^{T}$. In correspondence with [11], internal forces $\boldsymbol{f}_{\text {int }}$ lie in the null-space of the grasp matrix

$$
G=\left(G_{1}, G_{2}, \ldots, G_{N}\right) \quad \text { with } \quad G_{i}=I_{3}
$$

w.r.t all measured forces. In case of Cartesian positions, the grasp matrix is simply the identity matrix. The grasp matrix $G$ describes the relation of the forces of each robot frame and the object frame, and is well-established for robotic grasping and dexterous multi-fingered manipulation. As $G$ is not square, its Moore-Penrose pseudo-inverse

$$
G^{\dagger}=\left(G_{1}^{\dagger}, G_{2}^{\dagger}, \ldots, G_{N}^{\dagger}\right)^{T} \quad \text { with } \quad G_{i}^{\dagger}=\frac{1}{N} I_{3}
$$

is used for the null-space calculation of

$$
\boldsymbol{f}_{\text {int }}^{o}=\left[I-G^{\dagger} G\right] \boldsymbol{f}^{o} .
$$

For the sake of clarity we make the following assumptions.

Assumption 2: As we will be mainly concerned with the tracking performance in this paper, we assume that at the initial time $t_{0}$ all current and desired positions are equal, $\boldsymbol{\xi}_{i}\left(t_{0}\right)=\boldsymbol{\xi}_{i, d}\left(t_{0}\right)$. Initial configurations $\boldsymbol{\xi}_{i, d}\left(t_{0}\right)$ are chosen reasonable to have no internal stress at the beginning. This is necessary since an internal stress at initial time leads to an induced movement of the robotic manipulators, which is undesired for maintaining formation.

Assumption 3: There is no external force, $\boldsymbol{f}_{\mathrm{ext}}=0$, and the exact dynamic model (3) is known and can be subtracted from $f_{i}$ as a feedforward term.

Assumption 4: For simplicity, we assume that the influence of damping $D_{i}$ and inertia $M_{i}$ are negligible for the internal forces in (6). The multi-robot team moves at moderate velocities as we assume here, that the distributed impedances are in a quasi-equilibrium state and act thereby primarily via its stiffness $K_{i}$. The influence of damping $D_{i}$ and inertia $M_{i}$ is negligible with this assumption, which strongly simplifies the cooperative robot model. 
With these assumptions the major part of each force $f_{i}^{o}$ in (6) arises from the difference between desired and current manipulator position. Then, a balance of forces due to the impedance model is approximated as

$$
\boldsymbol{f}_{i}^{o}=K_{i}\left(\boldsymbol{\xi}_{i, d}-\boldsymbol{\xi}_{i}\right),
$$

where terms involving $M_{i}$ and $D_{i}$ vanish due to the previously made assumptions. When we establish the internal force model, we obtain the $i$ th internal force partition by inserting (7) into (6) and evaluating the result row-wise as

$$
\boldsymbol{f}_{i, \mathrm{int}}=K_{i}\left(\boldsymbol{\xi}_{i, d}-\boldsymbol{\xi}_{i}\right)-R_{w}^{o} G_{i}^{\dagger} \sum_{j=1}^{n} G_{j} R_{o}^{w} K_{j}\left(\boldsymbol{\xi}_{j, d}-\boldsymbol{\xi}_{j}\right) .
$$

Note that for transformation of the forces to the world frame $\Sigma_{w}$, each agent is aware of all rotation matrices $R_{w}^{o}$ and $R_{o}^{w}$.

3) Cooperative Impedance Control: Connecting the results (1), (2), and (8) with Assumptions 2- 4, we obtain

$M_{i} \ddot{\boldsymbol{\xi}}_{i}+D_{i}\left(\dot{\boldsymbol{\xi}}_{i}-\dot{\boldsymbol{\xi}}_{i, d}\right)+K_{i}\left(\boldsymbol{\xi}_{i}-\int_{t_{0}}^{t_{e}} \dot{\boldsymbol{\xi}}_{i, d} \mathrm{~d} \tau\right)=\boldsymbol{f}_{i, d}-$

$K_{i}\left(\int_{t_{0}}^{t_{e}} \dot{\boldsymbol{\xi}}_{i, d} \mathrm{~d} \tau-\boldsymbol{\xi}_{i}\right)+R_{w}^{o} G_{i}^{\dagger} \sum_{j=1}^{n} G_{j} R_{o}^{w} K_{j}\left(\int_{t_{0}}^{t_{e}} \dot{\boldsymbol{\xi}}_{j, d} \mathrm{~d} \tau-\boldsymbol{\xi}_{j}\right)$.

Remark 1: In most impedance control models a new state is introduced for $\left(\int_{t_{0}}^{t_{e}} \dot{\boldsymbol{\xi}}_{j, d} \mathrm{~d} \tau-\boldsymbol{\xi}_{j}\right)$ in order to reduce the degree of the system. For cooperative manipulation, we cannot apply this argument because the minimal state space representation involves both absolute and relative positions. Therefore, the system degree of one manipulator remains 3 .

4) State Space Model: Let $\boldsymbol{x}_{i}=\left[\left(\int_{t_{0}}^{t} \dot{\boldsymbol{\xi}}_{i, d} \mathrm{~d} \tau\right)^{T}, \boldsymbol{\xi}_{i}^{T}, \dot{\boldsymbol{\xi}}_{i}^{T}\right]^{T}$ be the system state and $\boldsymbol{u}_{i}=\left[\dot{\boldsymbol{\xi}}_{i, d}^{T}, \boldsymbol{f}_{i, d}^{T}\right]^{T}$ be the control input. Then a state space model for a single manipulator in cooperation results in

$$
\dot{\boldsymbol{x}}_{i}=A_{i i} \boldsymbol{x}_{i}+B_{i i} \boldsymbol{u}_{i}+\sum_{j \in\{1, \ldots, N\} \backslash\{i\}} A_{i j} \boldsymbol{x}_{j},
$$

with

$$
\begin{gathered}
A_{i i}=\left(\begin{array}{ccc}
0 & 0 & 0 \\
0 & 0 & 1 \\
\frac{1}{N} M_{i}^{-1} K_{i} & -\frac{1}{N} M_{i}^{-1} K_{i} & -M_{i}^{-1} D_{i}
\end{array}\right) \\
A_{i j}=M_{i}^{-1} R_{w}^{o} G_{i}^{\dagger}\left(\begin{array}{ccc}
0 & 0 & 0 \\
0 & 0 & 0 \\
G_{j} R_{o}^{w} K_{j} & -G_{j} R_{o}^{w} K_{j} & 0
\end{array}\right) \\
B_{i i}=\left(\begin{array}{cc}
1 & 0 \\
0 & 0 \\
M_{i}^{-1} D_{i} & M_{i}^{-1}
\end{array}\right),
\end{gathered}
$$

where $A_{i i}$ is the system matrix of a single manipulator $i, B_{i i}$ is its input matrix, and $A_{i j}$ represents the physical coupling from manipulator $j$ to manipulator $i$.

After deriving the system dynamics for a multi-robot cooperative system, we obtain a standard LTI sytem.

$$
\dot{\boldsymbol{x}}=A \boldsymbol{x}+B \boldsymbol{u}
$$

with the aggregated state vector $\boldsymbol{x}=\left[\boldsymbol{x}_{1}^{T}, \ldots, \boldsymbol{x}_{N}^{T}\right]^{T}$ and the aggregated input vector $\boldsymbol{u}=\left[\boldsymbol{u}_{1}^{T}, \ldots, \boldsymbol{u}_{N}^{T}\right]^{T}$. The total system is written as $A=\left[A_{i j}\right]$ and $B=\operatorname{diag}\left(B_{11}, \ldots, B_{N N}\right)$.

\section{B. Formation rigidity in multi-robot cooperation}

In this section we describe the control goal considered in this paper. We want to design a linear state feedback controller $\boldsymbol{u}=-K \boldsymbol{x}$ which optimally drives a formation of interconnected manipulators described by the system dynamics (14) from an initial condition $\boldsymbol{x}_{0}$ to a desired end point $\boldsymbol{x}_{e}$ while maintaining the initial formation. In the following we describe the cost functional of our LQR-like optimal control problem and how the desired rigidity is relaxed.

In order to achieve goal regulation to the goal $\boldsymbol{x}_{e}$, we employ the standard transformation of our state $\boldsymbol{x}$ into

$$
\tilde{\boldsymbol{x}}=\boldsymbol{x}-\boldsymbol{x}_{e} .
$$

Then, we can formulate the LQR cost functional which gives a controller driving to the end point in an optimal fashion as

$$
J=\tilde{\boldsymbol{x}}^{T}(T) S \tilde{\boldsymbol{x}}(T)+\int_{0}^{T} \tilde{\boldsymbol{x}}^{T}(t) Q \tilde{\boldsymbol{x}}(t)+\boldsymbol{u}^{T}(t) R \boldsymbol{u}(t) \mathrm{d} t,
$$

where $S, Q$ (positive semi-definite), and $R$ (positive definite) are weighting matrices expressing the desired performance. The weighting matrices need to be positive (semi-)definite in order to guarantee a minimum with the corresponding minimum cost finitely bounded from below.

Next, we define rigidity of the formation and how we can integrate rigidity into the cost functional. The formation is described by a static set of edges $E$ with cardinality $\|E\|$ between the manipulators such that the virtual structure of the formation is rigid during the movement phase. and keep their formation regardless of any direct mechanical coupling. Rigidity of the formation is described by an edge function $f(\boldsymbol{x})=\left(\ldots,\left\|\boldsymbol{\xi}_{i}-\boldsymbol{\xi}_{j}\right\|, \ldots\right) \in \mathbb{R}^{\|E\|}$ which is required to satisfy $f(\boldsymbol{x})=\boldsymbol{p}$. Identical to (10) and (14), the state variable $\boldsymbol{x}$ is concenated by $\boldsymbol{x}_{i}$ and also contains the manipulator positions $\boldsymbol{\xi}_{i}$. Here, $\boldsymbol{p}=\left(\ldots, \boldsymbol{p}_{i j}, \ldots\right)$ is the desired rigid distance between two manipulators, and constant if rigidity is achieved. Differentiating $f$ w.r.t time leads to

$$
\left(\boldsymbol{\xi}_{i}-\boldsymbol{\xi}_{j}\right)^{T}\left(\dot{\boldsymbol{\xi}}_{i}-\dot{\boldsymbol{\xi}}_{j}\right)=0 . \quad \forall(i, j) \in E
$$

The geometrical interpretation of (17) is that the difference in position between two linked manipulators is orthogonal to the difference in velocity. This equation represents our second control goal of maintaining formation rigidity. Due to Assumption 2 it is sufficient to maintain the formation instead of establishing it. In order to include the rigidity 
condition (17) into our LQR cost functional (16), we transform it into a quadratic term of the states. Thus (17) is written as $\boldsymbol{x}_{i, j}^{T} Q_{i j} \boldsymbol{x}_{i, j}$ with $\boldsymbol{x}_{i, j}=\left[\boldsymbol{x}_{i}^{T}, \boldsymbol{x}_{j}^{T}\right]^{T}$ by defining the blocks

$$
\begin{aligned}
& {\left[q_{i i}\right]=\left(\begin{array}{ccc}
0_{n \times n} & 0_{n \times n} & 0_{n \times n} \\
0_{n \times n} & 0_{n \times n} & \frac{1}{2} I_{n} \\
0_{n \times n} & \frac{1}{2} I_{n} & 0_{n \times n}
\end{array}\right) \forall(i, j) \in E} \\
& {\left[q_{i j}\right]=\left[q_{j i}\right]=\left(\begin{array}{ccc}
0_{n \times n} & 0_{n \times n} & 0_{n \times n} \\
0_{n \times n} & 0_{n \times n} & -\frac{1}{2} I_{n} \\
0_{n \times n} & -\frac{1}{2} I_{n} & 0_{n \times n}
\end{array}\right) \forall(i, j) \in E}
\end{aligned}
$$

The resultant matrix $Q_{i j}=\left[\begin{array}{cc}q_{i i} & q_{i j} \\ q_{j i} & q_{j j}\end{array}\right]$ is symmetric but indefinite and thus it cannot be employed in a standard LQR problem directly. Since the equality constraint described in Eq. (17) can be violated in both directions, the indefiniteness of $\boldsymbol{x}_{i, j}^{T} Q_{i j} \boldsymbol{x}_{i, j}$ is obvious, and its apparent global minimum is $-\infty$. The biquadratic term $\left(\boldsymbol{x}_{i, j}^{T} Q_{i j} \boldsymbol{x}_{i, j}\right)^{2}$ on the other hand has a minimum of 0 , and is thus suitable to be included in an optimization to ensure relaxed rigidity. In other words, minimizing $\left(\boldsymbol{x}_{i, j}^{T} Q_{i j} \boldsymbol{x}_{i, j}\right)^{2}$ for all $(i, j) \in E$ relaxes the equality constraint (17) into a minimization problem. Relaxation means that the resulting controller does not guarantee exact satisfaction of Eq. (17) for all times, but for appropriate weighting matrices, the controller design leads to values that are at least close to zero. Proper partitioning allows writing $\left(\boldsymbol{x}_{i, j}^{T} Q_{i j} \boldsymbol{x}_{i, j}\right)^{2}$ as

$$
\left(\boldsymbol{x}^{T} Q_{k} \boldsymbol{x}\right)^{2} \forall k \in\{1, \ldots,\|E\|\} .
$$

While the control design goal of goal regulation requires the transformation to the coordinates $\tilde{\boldsymbol{x}}$ from (15), it is important to note that the relaxed rigidity condition (19) still needs to be satisfied in the original coordinate system $x$. In order to combine both coordinate systems in the same cost functional we introduce an extended state vector

$$
\overline{\boldsymbol{x}}=\left(\tilde{\boldsymbol{x}}^{T}, 1\right)^{T} .
$$

With this state vector, we reformulate the relaxed rigidity condition (19) into

$$
\begin{aligned}
& \left(\boldsymbol{x}^{T} Q_{k} \boldsymbol{x}\right)^{2} \forall k \in\{1, \ldots,\|E\|\} \\
= & \left(\left(\begin{array}{c}
\tilde{\boldsymbol{x}} \\
1
\end{array}\right)^{T}\left(\begin{array}{cc}
Q_{k} & Q_{k} \boldsymbol{x}_{e} \\
\boldsymbol{x}_{e}^{T} Q_{k} & \boldsymbol{x}_{e}^{T} Q_{k} \boldsymbol{x}_{e}
\end{array}\right)\left(\begin{array}{c}
\tilde{\boldsymbol{x}} \\
1
\end{array}\right)\right)^{2} \\
= & \left(\overline{\boldsymbol{x}}^{T} \bar{Q}_{k} \overline{\boldsymbol{x}}\right)^{2} \forall k \in\{1, \ldots,\|E\|\}
\end{aligned}
$$

We can now combine all of the terms into one cost functional and restate our control goal. The goal of our optimal control problem is to find a controller $\boldsymbol{u}=-\bar{K} \overline{\boldsymbol{x}}$ with structure $\bar{K}=\left[K, 0_{2 n N \times 1}\right]$ in order to minimize the following cost functional

$$
\begin{aligned}
J=\overline{\boldsymbol{x}}(T)^{T} \bar{S} \overline{\boldsymbol{x}}(T)+ & \int_{0}^{T} \sum_{k=1}^{\|E\|}\left(\overline{\boldsymbol{x}}^{T}(t) \overline{q_{k}} \bar{Q}_{k} \overline{\boldsymbol{x}}(t)\right)^{2} \\
& +\boldsymbol{u}^{T}(t) R \boldsymbol{u}(t)+\overline{\boldsymbol{x}}^{T} \bar{Q} \overline{\boldsymbol{x}} \mathrm{d} t,
\end{aligned}
$$

where $\bar{S}$ and $\bar{Q}$ have the structure $\bar{S}=\operatorname{diag}(S, 0)$ and $\bar{Q}=$ $\operatorname{diag}(Q, 0)$ in order not to penalize the additional 1-state, $\bar{Q}_{k}$ is given in (21) and $\bar{q}_{k}$ is a positive scalar weighting

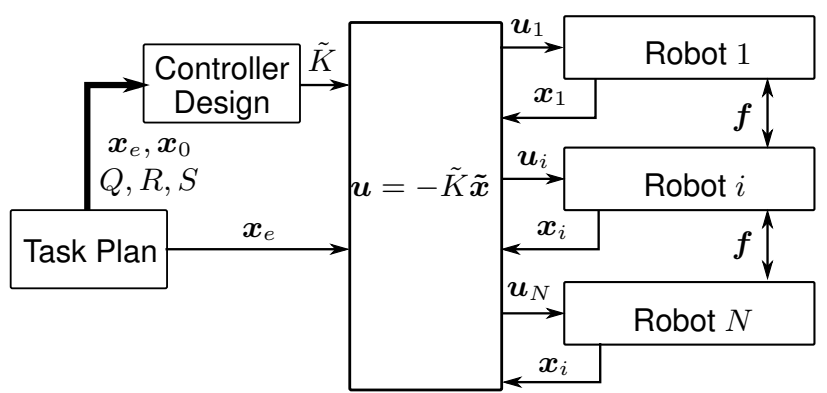

Fig. 2. Schematic overview of the cooperative mobile manipulation control architecture

factor. The term $\overline{\boldsymbol{x}}(T)^{T} \overline{\boldsymbol{S}} \overline{\boldsymbol{x}}(T)$ represents the penalty term resulting from the distance between $\boldsymbol{x}$ and $\boldsymbol{x}_{e}$ for the final time $T$. Control input constraints are indirectly realized by $\boldsymbol{u}^{T}(t) R \boldsymbol{u}(t)$. This cost functional represents our combined control goals of maintained formation by the term $\sum_{k=1}^{\|E\| \mid}\left(\overline{\boldsymbol{x}}^{T}(t) \bar{Q}_{k} \overline{\boldsymbol{x}}(t)\right)^{2}$, and goal regulation by the term $\overline{\boldsymbol{x}}^{T} \bar{Q} \overline{\boldsymbol{x}}$. The zero column in $\bar{K}$ is necessary to discard the augmented 1-state.

\section{LQR-LIKE OPTIMAL CONTROL}

When driving a multi-robot team from an initial configuration to a desired final configuration, a controller approach is depicted in Fig. 2. A task plan is obtained from a supervisory high-level instance, e.g. a human operator commanding the goal. Here, the task plan involves an initial configuration $\boldsymbol{x}_{0}$ and a final configuration $\boldsymbol{x}_{e}$ of the multi-robot team.

While there is a linear relationship in the standard LQR problem between the primal states $\boldsymbol{x}$ and the adjoint states $\boldsymbol{\lambda}$ given by $\boldsymbol{\lambda}(t)=P \boldsymbol{x}(t)$, allowing for the solution to use a Riccati equation for the matrix $P$, this is not the case here. Therefore we present an iterative descent algorithm to find a (local) minimum of the biquadratic cost functional (22) using adjoint states. The algorithm is inspired by the results in [9] and due to the non-convexity of the optimization problem, the result may be only suboptimal.

The corresponding Lagrangian function of the problem is

$$
\begin{aligned}
L= & \overline{\boldsymbol{x}}(T)^{T} \bar{S} \overline{\boldsymbol{x}}(T)+\int_{0}^{T} \sum_{k=1}^{\|E\|}\left(\overline{\boldsymbol{x}}^{T}(t) \bar{q}_{k} \bar{Q}_{k} \overline{\boldsymbol{x}}(t)\right)^{2} \\
& +\overline{\boldsymbol{x}}^{T}(t) \bar{K}^{T} R \bar{K} \overline{\boldsymbol{x}}(t)+\overline{\boldsymbol{x}}^{T} \bar{Q} \overline{\boldsymbol{x}} \\
& +\overline{\boldsymbol{\lambda}}^{T}(t)(\dot{\overline{\boldsymbol{x}}}(t)-(\bar{A}-\bar{B} \bar{K}) \overline{\boldsymbol{x}}(t)) \mathrm{d} t+\overline{\boldsymbol{\mu}}\left(\overline{\boldsymbol{x}}(0)-\overline{\boldsymbol{x}}_{0}\right),
\end{aligned}
$$

where $\bar{A}=\operatorname{diag}(A, 0)$ and $\bar{B}=\left[B^{T}, 0_{1 \times m}^{T}\right]^{T}$.

Partial integration of (23) gives

$$
\begin{aligned}
L= & \overline{\boldsymbol{x}}(T)^{T} \bar{S} \overline{\boldsymbol{x}}(T)+\int_{0}^{T} \sum_{k=1}^{\|E\|}\left(\overline{\boldsymbol{x}}^{T}(t) \bar{q}_{k} \bar{Q}_{k} \overline{\boldsymbol{x}}(t)\right)^{2} \\
& +\overline{\boldsymbol{x}}^{T}(t) \bar{K}^{T} R \bar{K} \overline{\boldsymbol{x}}(t)+\overline{\boldsymbol{x}}^{T} \bar{Q} \overline{\boldsymbol{x}} \\
& -\overline{\boldsymbol{x}}^{T}(t)(\bar{A}-\bar{B} \bar{K})^{T} \overline{\boldsymbol{\lambda}}(t)-\overline{\boldsymbol{x}}^{T}(t) \dot{\overline{\boldsymbol{\lambda}}}(t) \mathrm{d} t \\
& +\left[\overline{\boldsymbol{\lambda}}(t)^{T} \overline{\boldsymbol{x}}(t)\right]_{0}^{T}+\overline{\boldsymbol{\mu}}\left(\overline{\boldsymbol{x}}(0)-\overline{\boldsymbol{x}}_{0}\right),
\end{aligned}
$$


We can derive equations for the adjoint state through the optimality condition $\frac{\partial L}{\partial \overline{\boldsymbol{x}}}=0$. This gives

$$
\begin{aligned}
& \dot{\overline{\boldsymbol{\lambda}}}(t)=(\bar{A}-\bar{B} \bar{K})^{T} \overline{\boldsymbol{\lambda}}(t)-2 \bar{K}^{T} R \bar{K} \overline{\boldsymbol{x}}(t)-2 \bar{Q} \overline{\boldsymbol{x}}(t) \\
&-4 \sum_{k=1}^{\|E\|}\left(\overline{\boldsymbol{x}}^{T}(t) \bar{q}_{k} \bar{Q}_{k} \overline{\boldsymbol{x}}(t)\right) \bar{q}_{k} \bar{Q}_{k} \overline{\boldsymbol{x}}(t) \\
& \overline{\boldsymbol{\lambda}}(T)=-2 \bar{S} \overline{\boldsymbol{x}}(T), \quad \overline{\boldsymbol{\mu}}=-\overline{\boldsymbol{\lambda}}(0)
\end{aligned}
$$

The gradient is determined from the Lagrange function as

$$
\begin{aligned}
\left\langle L_{\bar{K}}, H\right\rangle & =\int_{0}^{T} 2 \overline{\boldsymbol{x}}^{T}(t) H^{T} R \bar{K} \overline{\boldsymbol{x}}(t)+\overline{\boldsymbol{x}}^{T}(t) H^{T} B^{T} \overline{\boldsymbol{\lambda}}(t) \mathrm{d} t \\
& =\int_{0}^{T} 2 R \bar{K} \overline{\boldsymbol{x}}(t) \overline{\boldsymbol{x}}^{T}(t)+B^{T} \overline{\boldsymbol{\lambda}}(t) \overline{\boldsymbol{x}}^{T}(t) \mathrm{d} t \bullet H
\end{aligned}
$$

where $H$ is a variation in $\bar{K}$, and where • denotes the Frobenius inner product. We use the gradient to iteratively reduce the cost. The step size used for the descent algorithm in each iteration step should satisfy the Wolfe conditions [12]

$$
\begin{aligned}
& J\left(\bar{K}+\gamma_{k} \operatorname{mat}\left(\boldsymbol{s}_{k}\right)\right)-J(\bar{K}) \leq \gamma_{k} c_{1}\left(\operatorname{vec}\left(\nabla_{\bar{K}} J\right)\right)^{T} \boldsymbol{s}_{k} \\
&\left(\operatorname{vec}\left(\nabla_{\bar{K}+\gamma_{k} \boldsymbol{s}_{k}} J\right)\right)^{T} \boldsymbol{s}_{k} \geq c_{2}\left(\operatorname{vec}\left(\nabla_{\bar{K}} J\right)\right)^{T} \boldsymbol{s}_{k},
\end{aligned}
$$

where $c_{1} \in(0,1)$ and $c_{2} \in\left(c_{1}, 1\right)$.

We determine the suboptimal feedback by using the following algorithm based on the quasi-Newton BroydenFletcher-Goldfarb-Shanno (BFGS) method [12]. A quasiNewton method uses an approximate of the Hessian matrix in addition to the gradient to determine the search direction in each iteration step. The BFGS method is a specific method to determine the Hessian approximate and has been shown to perform well in practice.

Algorithm 1:

1) Choose $c_{1}, c_{2} \in \mathbb{R}, \bar{K}_{0} \in \mathbb{R}^{m \times n N}$. Pick a symmetric positive definite matrix $D_{0} \in \mathbb{R}^{m n N \times m n N}$, e.g. $D_{0}=I_{m n N}$.

2) Compute the search direction $s_{k}$ as

$$
\boldsymbol{s}_{k}=-D_{k}\left(\operatorname{vec}\left(\nabla_{\bar{K}} J\right)\right)
$$

3) Compute the step size $\gamma_{k}$ according to (26).

4) Update the feedback matrix

$$
\bar{K}^{(k+1)}=\bar{K}^{(k)}+\gamma_{k} \operatorname{mat}\left(\boldsymbol{s}_{k}\right) .
$$

5) Set $\boldsymbol{p}_{k}=\operatorname{vec}\left(\bar{K}^{(k+1)}\right)-\operatorname{vec}\left(\bar{K}^{(k)}\right)$ and $\boldsymbol{q}_{k}=$ $\left(\operatorname{vec}\left(\nabla_{\bar{K}^{(k+1)}} J\right)\right)-\left(\operatorname{vec}\left(\nabla_{\bar{K}^{(k)}} J\right)\right)$. Update the Hessian approximate as

$$
\begin{aligned}
D_{k+1}=D_{k} & +\frac{\left(\boldsymbol{p}_{k}-D_{k} \boldsymbol{q}_{k}\right) \boldsymbol{p}_{k}^{T}+\boldsymbol{p}_{k}\left(\boldsymbol{p}_{k}-D_{k} \boldsymbol{q}_{k}\right)^{T}}{\boldsymbol{p}_{k}^{T} \boldsymbol{q}_{k}} \\
& -\frac{\left(\boldsymbol{p}_{k}-D_{k} \boldsymbol{q}_{k}\right)^{T} \boldsymbol{q}_{k}}{\left(\boldsymbol{p}_{k}^{T} \boldsymbol{q}_{k}\right)^{2}} p_{k} p_{k}^{T}
\end{aligned}
$$

Note that the resulting feedback matrices of Algorithm 1 are optimized with respect to one specific initial condition $x_{0}$. In practice the initial condition might not be known during the control design stage, or might be slightly disturbed from the assumed one. As a solution we propose to average over several initial conditions for the simulated trajectories to obtain a controller that performs well for a set of starting points. The algorithm remains unchanged except for the gradient which is now given by

$$
\begin{array}{r}
\nabla_{\bar{K}} J=\frac{1}{n_{\text {samples }}}\left(\sum_{i=1}^{n_{\text {samples }}} \int_{0}^{T} 2 R \bar{K} \overline{\boldsymbol{x}}_{i}(t) \overline{\boldsymbol{x}}_{i}^{T}(t)\right. \\
\left.+B^{T} \overline{\boldsymbol{\lambda}}_{i}(t) \overline{\boldsymbol{x}}_{i}^{T}(t) \mathrm{d} t\right),
\end{array}
$$

where $\overline{\boldsymbol{x}}_{i}(t)$ and $\overline{\boldsymbol{\lambda}}_{i}(t)$ are the trajectories resulting from the $i$ th initial condition, and $n_{\text {samples }}$ are their total number, see [9] for more details on the iterative control design.

\section{EXPERIMENTS}

To evaluate the control performance and to demonstrate the applicability of the proposed control scheme, a full-scale experiment is conducted. Our presented results show the benefits of the proposed controller approach by variations of the initial conditions and its reduction of internal forces.

\section{A. Experimental Setup}

The experimental setup consists of two commercially available KUKA LWR 4+ (light-weight robot), which are mounted on a mobile platform. Since both manipulators are assembled on top of the same mobile platform, we circumvent the challenge of distributed robotic base frames, kinematic uncertainties, and communication uncertainties such as time delay and packet loss. Furthermore, a 6 DOF force/torque sensor $(J R 3)$ is mounted at the wrist of each manipulator in order to ensure manipulator configuration independent measurements. The Fast Research Interface (FRI) provided by $K U K A$ is embedded in MATLAB/Simulink in compliance with the existing software architecture which features a data-driven architecture built upon a real-time capable database [13]. It runs in a real-time control loop at a frequency of $1 \mathrm{kHz}$ ensured by the PREEMPT_RT Linux real time kernel patch and MATLAB Simulink Coder.

A Cartesian impedance (1) is implemented and then both measured forces and $M_{i}, D_{i}$, and $K_{i}$ are rotated into the world frame $\Sigma_{w}$. Both end-effector's Cartesian positions are captured by a QualiSys motion capture system at a frequency of $350 \mathrm{~Hz}$ from which we also obtain the transformation $R_{w}^{o}$ for (12). A workspace extension of both robotic manipulators is accomplished according to the approach presented in [2]

\section{B. Experimental Design}

Due to the decoupling of linear motions, we only consider translational motions for each manipulator. An unactuated revolute joint along yaw is mounted on each end-effector, such that the whole system can rotate while the manipulators move translational. Rotational motion of a manipulator hold the object orthogonal to its boundary for collision avoidance between manipulator and object.

To satisfy Assumption 1, the impedance parameters are set to $M_{1,2}=10 I_{3}, D_{1,2}=80 I_{3}$, 


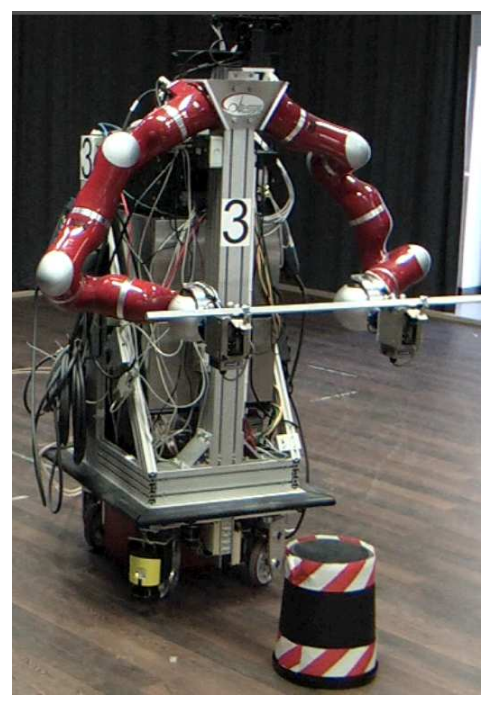

Fig. 3. Two robotic manipulators holding a bar and reaching a given goal configuration, whose centroid projection on the ground is marked by a striped object. Note that the two manipulators are independently controlled; the mounting on a single mobile platform prevents effects from kinematic and calibration uncertainties in the experiments.

and $K_{1,2}=120 I_{3}$ for both manipulators. Five possible initial values are chosen around $\boldsymbol{x}_{0}=$ $[1.769,2.08,1,1.769,2.08,1,0,0,0,1.769,1.52,1,1.769$, $1.52,1,0,0,0]^{T}$, with the relative manipulator distance $0.56 \mathrm{~m}$. The goal configuration for both manipulators is set in accordance with Assumption 2 to $\boldsymbol{x}_{e}=$ $[-1.08,-0.95,1,-1.08,-0.95,1,0,0,0,-0.52,-0.95,1$, - $0.52,-0.95,1,0,0,0]^{T}$, and the manipulaotrs at the final configuration are depicted in Fig. 3. The optimization parameters are chosen as $\bar{q}_{k}=1000, Q=0.01 I_{18}, R=$ $20 I_{18}$, and $S=1 I_{18}$.

Measurement of the system states for the feedback control law $\boldsymbol{u}=-\bar{K} \overline{\boldsymbol{x}}$ is performed such that for $\int_{t_{0}}^{t} \dot{\boldsymbol{\xi}}_{i, d} \mathrm{~d} \tau, \dot{\boldsymbol{\xi}}_{i, d}$ is integrated in the controller and initial value $\dot{\boldsymbol{\xi}}_{i, d}\left(t_{0}\right)$ is defined by the motion capture system. The manipulator endeffector position $\boldsymbol{\xi}_{i}$ is captured and $\dot{\boldsymbol{\xi}}_{i}$ is its time derivative.

With these parameters, we conduct an experiment in our laboratory and compare the results to a controller derived by the standard LQR implementation lqr of MATLAB/Simulink with the weighting matrizes $Q=1, R=1000$ in accordance with cost function (16).

\section{Results: Manipulators without Rigid Link}

In the first experiment, the robotic manipulators are driven to the final configuration without any rigid object in between the manipulators. In this scenario, we want to investigate if the multi-robot team maintains the formation in the experiment. Fig. 4 on the right shows the manipulator path from top view. The multi-robot team controller under rigidity relaxation according to (22) in a full-scale experiment is depicted in blue solid, the multi-robot team controller under LQR-like rigidity relaxation in simulation is depicted in green dashed, and the standard LQR controlled system ac- cording to (16) in red dashed-dotted. A good match between simulation and experiment is achieved in this setup. Both controllers regulate the multi-robot setup to the desired final configuration $\boldsymbol{x}_{e}$. However, half way to the goal there is a deviation between the rigidity-relaxed LQR-like and standard LQR. Our rigidity-relaxed controller approaches the goal configuration not in a direct way, but it maintains its formation. Since our controller takes the formation explicitly into account, it achieves far better results for multi-robot cooperation compared to a problem setup without rigidity constraints.

\section{Results: Manipulators with Rigid Link}

In addition to the previous results a rigid link in terms of a bar is fixed to the manipulators in order to evaluate the occurring internal forces. In Fig. 5 the LQR controlled system under rigidity relaxation according to (22) is depicted in solid blue line, the system controlled by standard LQR according to (16) is depicted in a dashed red line. A difference in both paths occurs, however it is not as strong as in the previous section due to the rigidity of the bar. However, the standard LQR controlled system is unable to attain the goal configuration. We assume that this is due to the rigid link which causes high effort in a rotation of the object.

Additionally, the internal force $\boldsymbol{f}_{i \text {,int }}$ along the rigid link, the only virtual linkage in a 2 robot team, is analyzed, see Fig. 5. Internal forces on the object are heavily reduced with a suboptimal controller under rigidity constraints. In the full-scale experiment, a light chattering of the robotic manipulator occurred due to a non-perfect following of the platform. We assume that this causes the formation violation between the two manipulator end-effector positions for the real-world scenario compared to the simulation results on the right side of Fig. 4.

\section{CONCLUSiOnS}

In this paper we propose an LQR-optimal control design for cooperative manipulation under rigidity constraints. This controller regulates the configuration of the multi-robot team and maintains its formation. To maintain the formation, the rigidity constraint is relaxed in the control design and results in a biquadratic term. Since a biquadratic term is unconventional, we have to rely on an iterative method to calculate the feedback matrix. The effectiveness and quality of the proposed controller is successfully demonstrated in experiments. For future work, we plan to decentralize the controller to more cooperating manipulators and investigate the chattering problem induced by the mobile platform.

\section{ACKNOWLEDGMENTS}

The work is partly supported by the German Research Foundation (DFG) excellence initiative research cluster "Cognition for Technical Systems CoTeSys", the European FP7 project (WEARHAP) and the DFG Priority Program SPP 1305 "Control Theory of Digitally Networked Dynamical Systems". 

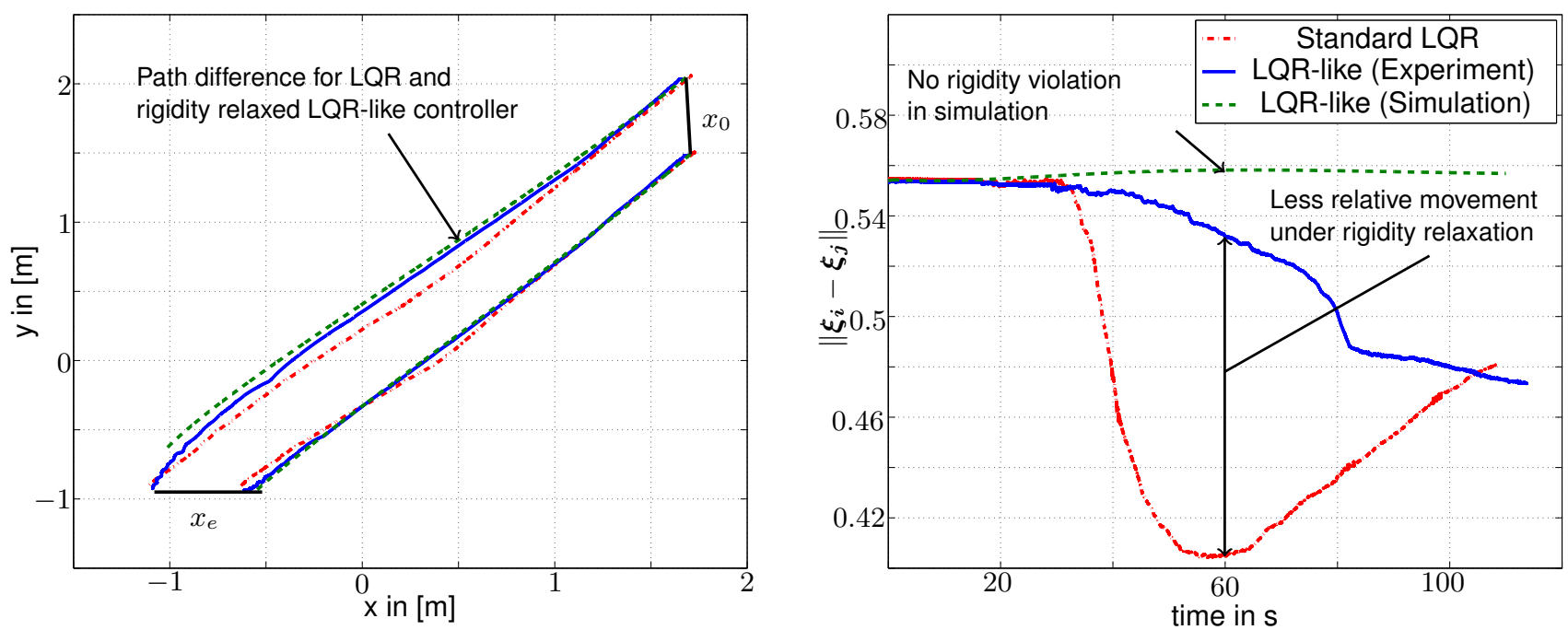

Fig. 4. Multi robot trajectories for standard LQR, biquadratic LQR-like problem in simulation and full-scale experiment without bar
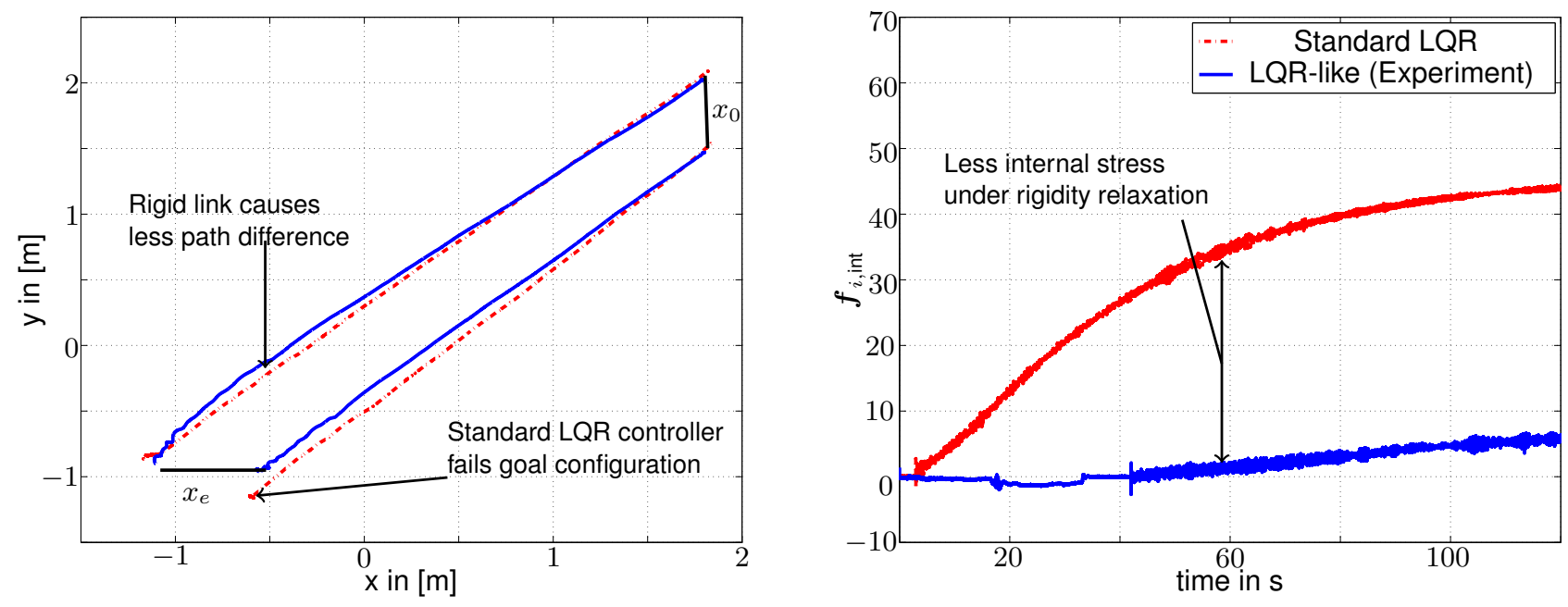

Fig. 5. Multi robot trajectories for standard LQR, biquadratic LQR-like problem in simulation and full-scale experiment with bar and internal force $\boldsymbol{f}_{i \text {,int }}$

\section{REFERENCES}

[1] J. Szewczyk, F. Plumet, and P. Bidaud, "Planning and controlling cooperating robots through distributed impedance," Journal of Robotic Systems, vol. 19, no. 6, pp. 283-297, 2002.

[2] S. Erhart, D. Sieber, and S. Hirche, "An impedance-based control architecture for multi-robot dual-arm mobile manipulation," in Proc. IEEE/RSJ IROS, 2013.

[3] M. Egerstedt and X. Hu, "Formation constrained multi-agent control," IEEE Trans. Robot. Autom, vol. 17, no. 6, pp. 947-951, 2001.

[4] M. Egerstedt, S. Martini, M. Cao, K. Camlibel, and A. Bicchi, "Interacting with networks: How does structure relate to controllability in single-leader, consensus networks?" IEEE Control Systems, vol. 32, no. 4, pp. 66-73, 2012.

[5] T. Eren, P. N. Belhumeur, B. D. Anderson, and A. S. Morse, "A framework for maintaining formations based on rigidity," in Proc. of the 15th IFAC World Congress, 2002, pp. 2752-2757.

[6] M. Cao, A. Morse, C. Yu, B. Anderson, and S. Dasgupta, "Maintaining a directed, triangular formation of mobile autonomous agents," Communications in Information and Systems, vol. 11, no. 1, p. 1, 2011.
[7] B. D. Anderson and J. B. Moore, Linear optimal control. PrenticeHall Englewood Cliffs, NJ, 1971, vol. 197, no. 1.

[8] K. Martensson and A. Rantzer, "Gradient methods for iterative distributed control synthesis," in Proc. IEEE/CSS CDC, 2009, pp. 549554

[9] F. Deroo, M. Ulbrich, B. D. O. Anderson, and S. Hirche, "Accelerated iterative distributed controller synthesis with a barzilai-borwein step size," in Proc. IEEE/CSS CDC, 2012, pp. 4864-4870.

[10] D. Sieber, F. Deroo, and S. Hirche, "Iterative optimal feedback controller design under relaxed rigidity constraints for cooperative manipulation,” 2013, proc. IEEE/CSS CDC (accepted).

[11] D. Williams and O. Khatib, "The virtual linkage: A model for internal forces in multi-grasp manipulation," in IEEE Int. Conf. on Robotics and Automation. IEEE, 1993, pp. 1025-1030.

[12] J. Nocedal and S. J. Wright, Numerical Optimization, 2nd ed. Springer, 2006.

[13] D. Althoff, O. Kourakos, M. Lawitzky, A. Mörtl, M. Rambow, F. Rohrmüller, D. Brščić, D. Wollherr, S. Hirche, and M. Buss, "An architecture for real-time control in multi-robot systems," Human Centered Robot Systems, pp. 43-52, 2009. 